\title{
The Effect of Product Positioning in a Comparison Table on Consumers' Evaluation of a Sponsor
}

Jungsil Choi

Cleveland State University, j.choi59@csuohio.edu

Duane W. Myer

Follow this and additional works at: https://engagedscholarship.csuohio.edu/bus_facpub

Part of the Advertising and Promotion Management Commons

How does access to this work benefit you? Let us know!

Publisher's Statement

"The final publication is available at Springer via http://dx.doi.org/[insert DOI]"."

Original Published Citation

Choi, J., Myer, D. W. (2012). The Effect of Product Positioning in a Comparison Table on Consumers' Evaluation of a Sponsor. Marketing Letters, 23, pp. 367-380.

This Article is brought to you for free and open access by the Monte Ahuja College of Business at EngagedScholarship@CSU. It has been accepted for inclusion in Business Faculty Publications by an authorized administrator of EngagedScholarship@CSU. For more information, please contact library.es@csuohio.edu. 


\title{
The effect of product positioning in a comparison table on consumers' evaluation of a sponsor
}

\author{
Jungsil Choi • Duane W. Myer
}

\begin{abstract}
This paper examines the effect on consumers' perceptions resulting from the positioning of a sponsor in relation to a competitor or competitors in a comparison table of a print advertisement. We hypothesize when a sponsor places its product in the right-hand panel and the competitor's product in the left-hand panel, respondents will react with a favorable evaluation for the sponsor as a result of two primary forces. The first is a general preference for an object positioned on the right as opposed to an object positioned on the left. The second is a vastly prevalent left-to-right reading habit. The prevalent left-to-right reading habit can lead consumers to subconsciously perceive this layout as a positive comparative advertisement, and the reversed placement is perceived as a negative comparative advertisement. We confirmed the hypothesis with three laboratory experiments.
\end{abstract}

Keywords Comparison advertisement - Comparison table · Physical positioning · Reading habit $\cdot$ Preference for right

\section{Introduction}

A comparison advertisement is one of the most common layouts used in both online and print advertising. A comparison advertisement is usually found in one of two primary forms. The first form features images of two or more products and its copy may or may not provide any specific information on the superiority of a sponsor. The second form is a more specific type of comparative advertisement that features a comparison table listing several specific features of each product, typically in a sideby-side comparison.

Comparative advertising has been the subject of a fair amount of research, yet the position of a sponsor in relation to a competitor in an advertisement featuring a 
comparison table has been largely overlooked. More specifically, there is limited evidence to support a template specifying what is the most effective positioning of the information within the advertisement. In support of such a template, we demonstrate that physical positioning of a sponsor in relation to a competitor in comparative advertising affects consumers' evaluation of the sponsor. The consumers' evaluation is attributed to two primary forces. The first is the general population's demonstrated preference for right versus left. The second is the prevalent left-to-right reading habit, which leads to a different valence of a comparative advertisement.

A review of online advertisements that featured comparison tables was conducted to support or reject the contention that a consistent template is used in the positioning of a sponsor's image, attributes, and claims. The search used the keywords "comparison table" and the results were narrowed by eliminating those advertisements comparing different versions of the same product, such as Tylenol and Tylenol PM. The remaining advertisements featuring a sponsor and a competitor supported the theory that a consistent template was used, and the ratio of left versus right positioning of the sponsor's product or service was 9 to 1 (please see Appendix A for details). Thus, the overwhelming majority of the advertisements placed a sponsor's image, attribute, or claim to the left of the competitor's; however, the question remains if this can be shown to be the most effective positioning of the sponsor. The goal of this paper is to provide convincing evidence to settle this debate by providing theoretical rationales and empirical support for physical positioning of a sponsor in a comparison table.

\section{Literature review}

\subsection{Preference for right versus left}

People show a preference for right versus left. Valenzuela and Raghubir (2009) have shown that people perceive both a higher price and higher quality for a product placed on the right-hand shelves than for a product placed on the left-hand shelves as a result of the tendency to presume that something on the right-hand side is superior at an unconscious or subconscious level. In a metaphorical sense, people perceive "right" as better than or bigger than "left" because the majority of people are right-handed. This implicit meaning of right emerges when it is consistent with physical positioning. For example, people tend to respond to large numbers faster on the right-hand side than on the left-hand side, and to small numbers on the left-hand side than on the right-hand side (Dehaene et al. 1993). Therefore, as they do with price and quality, people may unconsciously perceive something placed on the right-hand side as larger or better than the one placed on the left-hand side. That is why people respond more quickly as their expectancy is consistent with the setting.

A preference for right is also related to directionality, which is defined as the property of being directional or maintaining direction. For example, the directionality of the English language is from the left to the right. Left-to-right readers show a preference for stimuli with a rightward directionality because reading left-to-right corresponds to the direction of movement due to their natural reading habit (Chokron and Agostini 2000). Furthermore, directionality is related to a change of perceived status, such as low to high, current to improved, and worse to better. This phenomenon explains why most 
"before-and-after" type of comparative advertisements follow a consistent template in which a "before" example is positioned in the left-hand panel and an "after" example, which is an improvement over the "before" example, is positioned in the right-hand panel. Thus, left-to-right readers will have an expectation that an inferior example would be located on the left-hand side and an improved example on the right-hand side as this layout corresponds to a rightward directionality. This expectation could contribute to the formation of a preference for right versus left. Physical positioning of a sponsor and a competitor in a comparison table not only affects consumers' product preferences, but also may constitute two distinct types of comparative advertisements.

\subsection{A positive tone versus a negative tone in comparative advertisements} and the left-to-right reading habit

A comparative advertisement can be classified as either positive or negative based on the content or the tone of the advertisement's copy. A positive comparative advertisement focuses on a sponsor's advantages and benefits, but does so in such a manner so as not to derogate a competitor. A negative comparative advertisement tends to highlight a sponsor's advantages and benefits typically by casting dispersions on a competitor or by highlighting the imperfections of the competitor.

Previous studies have shown that a positive comparative advertisement results in more favorable attitudes toward a sponsor than does a negative comparative advertisement (Jain et al. 2006, 2007; Jain and Posavac 2004). Jain and Posavac (2004) suggest that the efficacy of a positive comparative advertisement versus a negative comparative advertisement can be attributed to a consumer's skepticism toward the negative messages, as well as the tendency to perceive the positive messages to be more believable. Negative messages induce more cognitive information processing than heuristic information processing, and relate to the elicitation of one's persuasion knowledge (Friestad and Wright 1994; Shiv et al. 1997). Therefore, people are more suspicious of the genuineness of negative messages than they are of positive messages, which, in turn, adversely affect the evaluation of a negative comparative advertisement.

Prior studies have shown that in a sequential observation of two objects, the later serves as the subject of comparison (the one being compared) and the object observed earlier serves as the referent of the comparison (the one providing the reference point) (Bruine de Bruin and Keren 2003; Mantel and Kardes 1999; Sanbonmatsu et al. 1991; Houston and Sherman 1995; Houston et al. 1989, 1991). Therefore, the expectation is that the information presented first should be the referent of comparison and the one presented later should be the subject. Extending this to a comparison table in an advertisement, we expect that the product placed in the left-hand panel should be the referent of comparison and the product placed in the right-hand panel should be the subject because people are likely to compare attributes in the right-hand panel back to attributes in the left-hand panel as a result of the tendency to read from left-to-right (Eviatar 1995). English readers tend to read print advertisements from left-to-right, which has been confirmed by tracking eye movements (Rayner et al. 2001). When a sponsor is placed in the left-hand panel and a competitor is placed in the right-hand panel, consumers are exposed to the features of the sponsor first, to which they compare the inferior features of the competitor. Those who are exposed to 
this configuration are likely to harbor such thoughts as "the competitor lacks certain qualities of the sponsor" or "the competitor is inferior in some aspects to the sponsor." These thoughts are also very similar to those evoked by a negative comparison advertisement. Conversely, if the physical positioning of the competitor and the sponsor is reversed, the opposite result is expected. Those who are exposed to the opposite configuration are likely to have such thoughts as "the sponsor is more appealing than the competitor" or "the sponsor is superior in some aspects to the competitor." Those thoughts are similar to those evoked by a positive comparison advertising message. Therefore, we hypothesize that consumers will have a more favorable attitude toward a sponsor when it appears in the right-hand panel of a comparison table, presumably because the positioning corresponds to a positive comparative advertisement.

Thus, our hypothesis that physical positioning of a sponsor in a comparison table will influence the effectiveness of a comparative advertisement is based on two theoretical rationales. The first is a preference for right versus left. The second is the perception of a positive versus negative comparative advertisement resulting from the left-to-right reading habit. Specifically, we hypothesize that consumers will attribute a greater perceived value for a sponsor when it is placed in the right-hand panel in a comparison table. Going forward, we will use the convention where "right-positioning" will refer to a comparative advertisement in which a sponsor is placed in the right-hand panel, and conversely "left-positioning" will refer to a comparative advertisement in which a sponsor is placed in the left-hand panel.

\section{Study 1}

In study 1, we demonstrated how respondents perceive the value of a sponsor differently as a result of its physical positioning in a comparison table. For example, when a generic brand (or a private label) is contrasted with an established leading brand, the advertisement emphasizes the generic brand's low price in conjunction with the claim of efficacy in relation to the leading brand. A comparison table can be highly efficient in these situations as it allows consumers to compare the attributes, such as the price, quantity, and the ingredients of the two products. In this setting, we hypothesize consumers will perceive a higher value for a generic product, which in turn will increase favorable attitudes toward the product when we place it in the right-hand panel in a comparison table.

H1: Consumers will attribute a greater perceived value for a sponsor, which in turn will increase the favorability toward the sponsor when placed in the right-hand panel versus the left-hand panel in a comparison table.

\subsection{Method}

\subsubsection{Stimuli}

We used the popular pain reliever Tylenol and a fictitious generic product named "Wonder" in the designed comparison advertisement. We chose a pain reliever as the stimulus because there are many store brands in this product category, so it is natural 
for respondents to observe these types of products in a comparison table. We placed Tylenol in the left-hand panel and Wonder in the right-hand panel in one stimulus and reversed the two products in the other stimulus. The comparison table provided information on five attributes: price (Wonder, \$2.99 vs. Tylenol, \$5.99), number of tablets (100 in both), active ingredients (acetaminophen $500 \mathrm{mg}$ in both), inactive ingredients (mostly identical, with a few minor differences), and FDA approval (both FDA approved). To prevent any confusion from the different inactive ingredients, it was clearly stated in a footnote that the inactive ingredients did not have a significant impact on the effectiveness of drugs. To minimize the likelihood of any distractions, no other features or stimuli other than the comparison table were included.

\subsubsection{Design and participants}

We used a single-factor (Wonder in the right-hand panel versus the left-hand panel) between-subjects design for the study. Seventy-seven college students from a large Midwestern university participated in the study for course credit.

\subsubsection{Procedure}

An experimenter described the purpose of the study as an effort to gather consumers' opinions about a generic drug. After the subjects viewed the comparative advertisement of Wonder and Tylenol, we asked them to evaluate Wonder against Tylenol. We measured the perceived value on Lin and Wang's (2006) three-item, nine-point scale (e.g., the advertised product is a very good value for the money/very poor for the money, $\alpha=0.87)$. We also measured attitudes toward Wonder on a three-item, nine-point scale $(\alpha=0.85)$.

\subsection{Results and discussion}

\subsubsection{The perceived value and attitudes toward the product}

We found a significant difference in the perceived value of Wonder, the sponsor depending on its physical positioning in the comparison table. We used the independent sample $t$ test to examine the difference in the perceived value and found when we placed Wonder in the right-hand panel of the comparison table, the respondents perceived a higher value for the brand $\left(M_{\text {right }}=8.38\right.$ vs. $M_{\text {left }}=7.73, t(75)=3.22$, $p<0.01)$. We also found that the respondents had more favorable attitudes toward Wonder when we placed it in the right-hand panel of the comparison table versus in the left-hand panel $\left(M_{\text {right }}=7.96\right.$ vs. $\left.M_{\text {left }}=7.46, t(75)=2.06, p<0.05\right)$.

\subsubsection{Mediation analysis}

We conducted a mediation analysis to assess if the perceived value mediates the effect of physical positioning and attitudes toward the sponsor. We tested the mediated relationship using Preacher and Hayes' (2004) SPSS INDIRECT Macro statistics package. We asked the package to generate 5,000 re-samples for bootstrapping and found the $95 \%$ bootstrap confidence interval $(\mathrm{CI})$ that does not include 0 (lower $\mathrm{CI}=0.106$ and upper 
$\mathrm{CI}=0.394)$. The results confirmed that the physical positioning affects the respondents' perceived value of Wonder, which in turn affects their attitudes toward the brand (Fig. 1).

Overall, the results were consistent with the hypothesis that the physical positioning of a sponsor in a comparison table affects respondents' evaluation of the product. In this study, we compared a sponsor to a single competitor in a comparative advertisement; however, comparative advertisements may also show a sponsor compared to several competitors. In the following study, we extend study 1 to the condition when multiple competitors were shown in contrast to a sponsor in a comparison table.

\section{Study 2}

The purpose of study 2 was twofold. One objective was to replicate study 1 's key findings that respondents have more favorable attitudes toward a sponsor when it is in the right-hand panel so as to increase the validity of the findings. The second objective was to extend the comparison context from a one competitor to multiple competitors to generalize the hypothesized impact of physical positioning in a comparative advertisement.

\subsection{Method}

\subsubsection{Stimuli}

We designed the stimulus by adopting an actual magazine advertisement for a 2010 Malibu LTZ and compared it to three competitors - a 2010 Toyota Camry XLE, a 2010 Honda Accord EX-L Sedan, and a 2010 Ford Fusion SEL. In an actual advertisement, the Malibu LTZ was placed in the far-left panel followed by the Camry, the Accord, and the Fusion, respectively. The comparison table in the advertisement listed six comparable features for each model, such as highway fuel economy, side-impact crash safety
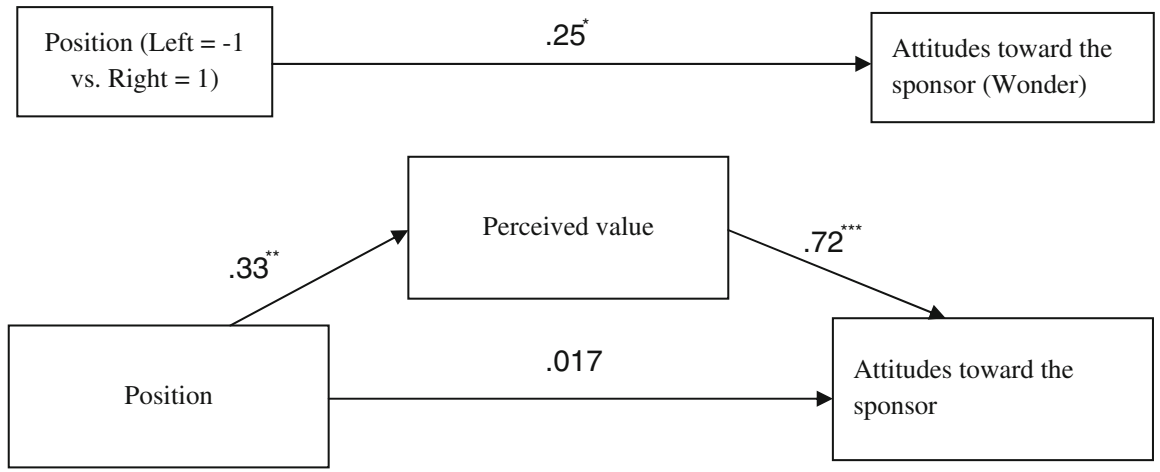

† Significant at the .1 level.

* Significant at the .05 level.

** Significant at the .01 level.

*** Significant at the .001 level.

Fig. 1 Mediated relation between physical positioning and attitudes toward the sponsor 
ratings, Insurance Institute for Highway Safety testing results, OnStar built-in automatic crash response, turn-by-turn navigation, and powertrain limited warranty. In addition, the advertisement included notes in the right-hand margin explaining each feature in detail. We used this advertisement for one stimulus after modifying it for this study by adding a photo of the product. We designed a second version of the stimulus by simply shifting the physical position of the Malibu LTZ to the far right in the comparison table of the advertisement.

\subsubsection{Design and participants}

We used a single-factor (position of the Malibu LTZ: in the far-right panel versus the far-left panel) between-subjects design for the study. Forty-one students from a major Midwestern university participated in the study for course credit. We excluded two respondents because they failed to complete the questionnaire.

\subsubsection{Procedure}

We randomly assigned participants to one of two treatment groups, and each group saw either the original comparison advertisement or the redesigned advertisement on a computer screen. They answered questions relating to the advertisement, after viewing their respective stimuli.

We measured attitudes toward the Malibu LTZ on a three-item, nine-point scale $(\alpha=0.94)$. We considered three variables as covariates. First, we measured attitudes toward the other competitors on the same scale $\left(\alpha_{\text {Camry }}=0.97, \alpha_{\text {Accord }}=0.97\right.$, $\left.\alpha_{\text {Fusion }}=0.94\right)$ because attitudes toward the other competitors may influence attitudes toward the Malibu LTZ. Secondly, we measured product familiarity on a one-item, five-point scale, because familiarity with the brand may affect a participant's evaluation of the product. Thirdly, we measured purchase relevance on a four-item, seven-point scale (i.e., important/unimportant, irrelevant/relevant, means nothing to me/means a lot to me, of concern to melof no concern to me; $\alpha=0.94)$.

Because we modified an original advertisement slightly by shifting the physical position of the Malibu LTZ to the far right, we needed to confirm that this modification did not affect perceived typicality of the advertisement. As a final step, we measured typicality of the advertisement on a two-item, seven-point scale $(\alpha=0.85)$ to prevent a counterargument that the impact of the physical positioning was attributed to advertisement typicality.

\subsection{Results and discussion}

\subsubsection{Attitudes toward the Malibu LTZ}

We found that all the variables being considered covariates were not correlated with the dependent variable, so we could not include them as covariates in the analyses (Keppel 1982). Thus, we used the independent sample $t$ test to assess the hypothesis. We found a significant difference in attitudes toward the Malibu LTZ in terms of its physical positioning in the comparison table of the advertisement $\left(M_{\text {far right }}=7.51\right.$, $\left.M_{\text {far left }}=6.33, t(37)=-2.63 ; p<0.02\right)$. As demonstrated by these measures, 
respondents evaluated the Malibu LTZ as more favorably when it was placed in the far-right panel in the comparison table as opposed to the far-left panel.

Furthermore, we could not find any significant difference in typicality between the two advertisements $\left(M_{\text {far right }}=5.14, M_{\text {far left }}=5.08, t(37)=-0.11, p>0.91\right)$, which rules out the possibility that the effect of physical positioning could be attributed to a lack of advertisement typicality. It was not deemed meaningful to attempt to measure the perceived value of the brand in this study where no information of price appears in the stimuli, because the perceived value has been shown to be largely estimated on the basis of pricing information. Furthermore, we considered a measurement of the perceived value redundant since we found a high correlation between the perceived value and attitudes toward the sponsor on the mediated relationship in study 1 .

\section{Study 3}

Studies 1 and 2 demonstrated that respondents expressed favorable attitudes toward the sponsor when placed in the right-hand panel as opposed to the left-hand panel. Of the two rationales discussed earlier, the preference for right versus left could be sufficient to explain the findings of these two studies. The second rationale is the prevalent left-toright reading habit, which is believed to bring about a different valence of a comparative advertisement. We hypothesized that right positioning evokes thoughts similar to those of a positive comparative advertisement and left positioning evokes thoughts similar to those of a negative comparative advertisement. In study 3, we investigate this hypothesis in conjunction with the tone of an advertisement copy. We assume if physical positioning elicits a different valence of a comparative advertisement, positive or negative, a complimentary message tone will enhance the effectiveness of the advertisement. For example, a positive message tone (e.g., "Our brand is better in some aspects than this competitive brand.") will enhance the effectiveness of right positioning. We theorize that this combination will be the most effective of the four possible combinations for the following reasons. First, respondents will perceive this combination of a positive copy and right positioning as being consistent in both layout and message. Therefore, we expect the consistency of physical positioning and tone will have a synergistic effect, and thus increase the effectiveness of the advertisement due to a preference for matching (Aaker and Lee 2006). Second, past research showed that a positive comparative advertisement is more effective than a negative comparative advertisement in enhancing attitudes toward a sponsor (Jain and Posavac 2004). This combination corresponds to a positive comparative advertisement.

Based on the matching preference, we expect the two non-matching combinations (i.e., a positive tone + left positioning and a negative tone + right positioning) will be less effective in enhancing attitudes toward a sponsor. Furthermore, we do not expect a negative message tone highlighting inferiority of a competitor (e.g., "This competitive brand is inferior is some respects to our brand.") will enhance the effectiveness of left positioning despite being consistent in both layout and message because this combination corresponds to a negative comparative advertisement. As mentioned above, a negative comparative advertisement is less effective in enhancing attitudes toward a sponsor than a positive comparative advertisement (Jain and Posavac 2004). Thus, we expect that this combination will also be less effective than the first 
matching combination. Therefore, of the four possible combinations, the combination of right positioning and a positive copy tone would be the most effective.

$\mathrm{H} 2$ : The right-positioning in combination with a positive copy tone will be considered the most amicable, as it corresponds to a positive comparative advertisement.

H3: Consumers will have the most favorable attitudes toward the sponsor with the combination of the right-positioning layout and a positive copy tone.

\subsection{Methods}

\subsubsection{Stimuli}

We used two Korean toothpaste brands (2080 and Median), neither of which are available in the US market, as the stimuli in the advertisement. Both brands provided information on four comparable attributes. The first was price, with the sponsor priced at $\$ 1.39$ and the competitor priced at $\$ 1.59$. Second, the sponsor had a whitening agent (hydrogen peroxide), whereas the competitor did not include a whitening agent. Third was the level of tooth decay protection and fourth was the duration of breath freshening. We manipulated the positive and negative comparison copies as follows:

The positive advertisement copy: 2080 (Median) is more effective in preventing tooth decay and promoting whitening than the leading brand, Median (2080).

The negative advertisement copy: The leading brand, 2080 (Median) is not as effective in preventing tooth decay and promoting whitening as Median (2080).

In a pretest, we checked typicality between two comparative advertisements using a positive and a negative tone. Thirty-eight college students participated in the study for course credit. We measured typicality on the same item utilized in study 2 between a positive and a negative tone, holding the physical positioning of the products constant. We did not find a significant difference in typicality in terms of a copy type $\left(M_{\text {positive }}=4.30\right.$ vs. $\left.M_{\text {negative }}=4.44, t(36)=-0.28, p>0.78\right)$.

\subsubsection{Design and participants}

We used a 2 (ad copy: positive vs. negative) $\times 2$ (position, left hand vs. right hand $) \times 2$ (sponsor's product name: Median vs. 2080) between-subjects design. One hundred ten students from a major Midwestern university participated in the study for course credit and they were randomly assigned to one of the eight conditions.

\subsubsection{Procedure}

Information provided to the participants about the sponsor described it as a newly launched toothpaste brand and described the competitor as a leading toothpaste brand in South Korea. An experimenter described the purpose of this survey as research to gauge consumers' preference and attitudes toward the two foreign toothpastes. We interchanged the product names to remove factors caused by an unforeseen name preference. 
We measured attitudes toward the sponsor on a three-item, nine-point scale $(\alpha=0.88)$. We also measured attitudes toward the advertisement on Jain and Posavac's (2004) five-item, seven-point scale $(\alpha=0.79)$ (i.e., the advertisement was hostile/ gentle to the competitor; the advertisement was critical/complementary the competitor; the advertisement was/was not derogatory of the competitor; the advertisement attempted to damage/did not attempt to damage the reputation of the competitor; the advertisement put down/praised the competitor). This new measurement was added in this study to verify whether respondents perceive an advertisement as more amicable in terms of the combinations of the ad copies and physical positioning of the comparison table in the advertisement. Finally, we asked participants if they were previously aware of these two toothpaste brands.

\subsection{Results and discussion}

None of the respondents in the study were familiar with either of the two brands, and we did not find any differences in any of the measured variables between the two products due to a name difference. Therefore, we combined the eight cells into four cells.

\subsubsection{Manipulation check}

It was necessary to check whether the respondents perceived the created positive and negative ad copy as each was intended. Thus, we expected that the positive copy to be perceived as more amicable than the negative copy. We found a marginal difference in perception of the positive and the negative copies when we controlled the impact of physical positioning $\left(M_{\text {positive }}=3.20, M_{\text {negative }}=2.89, t(108)=1.66 ; p<0.1\right)$. Participants reported that the positive copy was more amicable and less derogative toward the competitor.

\subsubsection{Valence for comparison}

A planned contrast showed that the respondents evaluated the combination of right positioning + a positive copy as more amicable than the other combinations (i.e., left positioning + a positive copy; right positioning + a negative copy; left positioning $+\mathrm{a}$ negative copy) $(t(106)=2.70, p<0.01)$. In other words, the respondents' perception of a positive comparative advertisement was the combination of right positioning and a positive copy, as this highlights the superiority of the sponsor without being offensive to the competitor. This confirmed hypothesis 2 . Table 1 provides specific statistics for individual $t$ test results.

\subsubsection{Attitudes toward the sponsor}

A planned contrast also showed that the combination of right positioning + a positive advertisement copy resulted in more favorable attitudes toward the sponsor than the other combinations $(t(106)=3.14, p<0.01)$, which confirmed hypothesis 3 . Because the right-positioning layout is perceived as a positive comparative advertisement, a positive ad copy is consistent with the layout. As a result, this matching enhances the effectiveness of the comparative advertisement. The results concerning attitudes 
Table 1 Evaluation for the sponsor in terms of a copy tone (positive vs. negative) and physical positioning (right vs. left)

\begin{tabular}{lcclcc}
\hline & Right+positive & Others & & $t$ value & $P$ value \\
\hline Valence for the ad & \multirow{2}{*}{3.45} & 2.93 & Left + positive & 2.33 & $<0.05$ \\
& & 2.96 & Left + negative & 2.12 & $<0.05$ \\
& & 2.85 & Right + negative & 2.24 & $<0.05$ \\
Attitudes toward the sponsor & \multirow{2}{*}{6.90} & 6.14 & Left + positive & 2.72 & $<0.01$ \\
& & 6.06 & Left + negative & 2.91 & $<0.01$ \\
& & 6.20 & Right + negative & 2.13 & $<0.05$ \\
\hline
\end{tabular}

toward the sponsor and the advertisement are consistent with the effectiveness of a positive comparative advertisement. Table 1 provides more specific $t$ test results for individual comparisons.

\section{General discussion}

We conducted three experiments on the effectiveness of comparative advertisements, hypothesizing that such advertisements would be more effective when a sponsor is positioned in the right-hand panel of a comparison table as opposed to the left-hand panel. In support of this hypothesis, we put forth two theoretical foundations as the rationale. The first was the expectation of a higher perceived value for a sponsor when positioned in the right-hand panel. This was due to the vast preference for right versus left. The second was the expectation that the respondents would perceive right positioning as a positive comparative advertisement. This was due to the widely prevailing left-to-right reading habit. In study 1 , the physical positioning of the sponsor resulted in a significantly higher perceived value and more favorable attitudes toward the sponsor. In study 2, the effects of physical positioning of the sponsor in relation to a single competitor were shown to be valid when extended to another common form of comparative advertisement in which the sponsor was compared to multiple competitors. Again, this showed positioning the sponsor in the far-right panel resulted in more favorable attitudes toward the sponsor. In study 3, the physical positioning was shown to affect the perception of the valence of comparison advertisements. When the sponsor was positioned in the right-hand panel and the advertisement's copy reflected a positive tone, respondents perceived it as more amicable than all the other combinations of positioning and copy tone. The combination of right positioning and a positive tone resulted in the highest measure of favorability toward the sponsor.

The findings of study 3 imply that the effect of physical positioning is attributed to more than just a preference for right versus left. If a preference for right versus left was the sole reason for the high level of favorability, the combination of right positioning and the negative copy tone should have demonstrated comparable effects when compared to the combination of right positioning and the positive copy tone. If the positive tone versus the negative tone of the advertisement by itself resulted in the observed difference, the combination of left positioning and the positive copy tone, as well as the combination of left positioning and the negative copy tone should have had a different 
effect. Therefore, these studies show that the effect of physical positioning results not only from a preference for the sponsor positioned in the right-hand panel, but also from the perception of a different valence of a comparative advertisement.

The results show that a positive comparative copy is not, by itself, sufficient to enhance the evaluation of a comparative advertisement, especially when this type of advertisement features a comparison table. When a copy type, such as positive versus negative, is in harmony with appropriate positioning in a comparison table, the effectiveness of a comparative advertisement improves significantly. These results suggest that a positive copy should be combined with a right-positioning layout. The findings in these studies have practical managerial implications. Marketing managers and advertisers now have evidence concerning the effect of physical positioning of a sponsor in a comparison table. This paper shows how this minor change results in significant differences in the effectiveness of a comparative advertisement. Furthermore, the findings provide empirical evidence that challenges the current phenomena in comparison tables. As we discussed earlier, approximately $90 \%$ of the comparison tables utilized a left-positioning template for a sponsor, although it has now been shown to be less effective in our studies than a right-positioning template. The findings provide guidelines for designing the most effective comparative advertisement by considering the physical positioning of a sponsor in relation to a competitor or competitors in a comparison table and the tone of an advertisement copy.

\subsection{Limitations and future study}

Logically, the next step would be to investigate this hypothesis where the subjects were selected from another cultural group who reads from right-to-left, such as Chinese participants; however, such an undertaking would be rather challenging. Morikawa and McBeath (1992) found that when perceiving movement of visual stimuli, readers with a left-to-right reading habit have a robust bias to experience leftward movement. In comparison, readers with a right-to-left reading habit have neither a bias to report leftward movement, nor a bias to report rightward movement. This is attributed to the latter groups' exposure to learning another language whose direction is left-to-right. As many people worldwide learn English, those with a right-to-left reading habit typically experience reading in both directions. Therefore, the effect of a right-to-left reading habit on the perception of movement of visual stimuli is attenuated. This is the primary reason for finding appropriate subjects across different linguistic systems is difficult, as many are already biased by an exposure to a left-to-right reading habit.

Although a preference for right versus left supports our hypotheses, it still requires more explorative research to investigate the preference for objects positioned on the right-hand side. Although there are some pieces of empirical evidence, theoretical rationales could not support the mechanism entirely. There are several metaphorical associations of right as something that is normal, positive, or superior. For example, the majority of people are right-handed. Thus, the right hand serves as the dominate hand and the left hand only plays a supplemental role. In addition, there are several dictionary definitions of right where most imply goodness, morally good, true, correct, normal, and complete. These learnt associations might be activated unconsciously and contribute to the formation of a preference for right whereby thinking something in the right-hand panel better. Therefore, it may be of 
interest to investigate the relation of metaphorical means of right and its functional implications in various marketing stimuli.

\section{Appendix A}

Table 2 The list of brands observed online

\begin{tabular}{|c|c|c|c|}
\hline $\begin{array}{l}\text { Company (brand) } \\
\text { sponsoring the ad }\end{array}$ & Company (brand) being compared & $\begin{array}{l}\text { Position of the } \\
\text { company (brand) } \\
\text { sponsoring the ad }\end{array}$ & Type of company \\
\hline Tradeking & OptionsXpress & Left & Online trading \\
\hline Blackberry Storm & iPhone $3 \mathrm{G}$ & Left & Mobile phone \\
\hline Samsung i780 & i-Mate 8502 & Left & Mobile phone \\
\hline Apple iPhone & $\begin{array}{l}\text { Nokia N95, Samsung Blackjack, } \\
\text { Blackberry Curve } 8300, \\
\text { and Palm } 740\end{array}$ & Left & Mobile phone \\
\hline SongSwap & Concert Vault & Left & Phone applications \\
\hline XpanD/Active 3D & Dolby & Left & $3 \mathrm{D}$ providers \\
\hline iFly Touch 3 Pad & Apple iPad & Left & Tables \\
\hline Advantix & Frontline plus & Left & Insecticide \\
\hline AVG Free & Max PC Secure & Left & Computer software \\
\hline Fonera 2.0 & $\begin{array}{l}\text { ASUS WL-500 G, Planex BRC-14VG, } \\
\text { D-Link DI-524UP, and the } \\
\text { other four competitors }\end{array}$ & Left & Network device \\
\hline Fiberglass composite & $\begin{array}{l}\text { Aluminum with Thermal Break } \\
\text { and Vinyl }\end{array}$ & Left & Material \\
\hline Wattson & Efergy, Eco-Eye, and OWL & Left & Energy monitors \\
\hline XP-Mode & Zinstall & Right & PC solutions \\
\hline VMware View 4 & $\begin{array}{l}\text { Citrix XenDesktop 4, Microsoft RDS, } \\
\text { and Provision Network Wrokspace }\end{array}$ & Left & PC solutions \\
\hline MasterMine & $\begin{array}{l}\text { Crystal Reports and GoldMine } \\
\text { Report Writer }\end{array}$ & Left & Computer software \\
\hline PlayStation Move & Xbox Kinect and Nintendo Wii & Left & Games \\
\hline IS-Jobs & TES, IBO, TIE Online & Left & Online job search \\
\hline Reimage Repair tool & $\begin{array}{l}\text { Online Technician, Anti-virus Packages, } \\
\text { and Registry Cleaner Solutions }\end{array}$ & Left & PC solutions \\
\hline Invisalign & Fixed Braces & Left & Dental services \\
\hline Accredited & Licensed & Right & Plumbers training \\
\hline
\end{tabular}

\section{References}

Aaker, J. L., \& Lee, A. Y. (2006). Understanding regulatory fit. Journal of Marketing Research, 43 (1), 15-19.

Bruine de Bruin, W., \& Keren, G. (2003). Order effects in sequentially judged options due to the direction of comparison. Organizational Behavior and Human Decision Processes, 92(1-2), 91-101. 
Chokron, S., \& Agostini, M. De. (2000). Reading habits influence aesthetic preference. Cognitive Brain Research, 10(2), 45-49.

Dehaene, S., Serge, B., \& Giraux, P. (1993). The mental representation of parity and number magnitude. Journal of Experimental Psychology. General, 122(3), 371-396.

Eviatar, Z. (1995). Reading direction and attention - effects on lateralized ignoring. Brain and Cognition, 29(2), 137-150.

Friestad, M., \& Wright, P. (1994). The persuasion knowledge model: How people cope with persuasion attempts. Journal of Consumer Research, 21(1), 1-31.

Houston, D. A., \& Sherman, S. J. (1995). Cancellation and focus: The role of shared and unique features in the choice process. Journal of Experimental Social Psychology, 31(4), 357-378.

Houston, D. A., Sherman, S. J., \& Baker, S. M. (1989). The influence of unique features and direction of comparison of preferences. Journal of Experimental Social Psychology, 25(2), 121-141.

Houston, D. A., Sherman, S. J., \& Baker, S. M. (1991). Feature matching, unique features, and the dynamics of the choice process: Predecision conflict and postdecision satisfaction. Journal of Experimental Social Psychology, 27(5), 411-430.

Jain, S. P., \& Posavac, S. S. (2004). Valenced comparisons. Journal of Marketing Research, 41(1), 46-58.

Jain, S. P., Agrawal, N., \& Maheswaran, D. (2006). When more may be less: The effects of regulatory focus on different comparative frames. Journal of Consumer Research, 33(1), 91-98.

Jain, S. P., Lindsey, C., Agrawal, N., \& Maheswaran, D. (2007). For better or for worse? Valenced comparative frames and regulatory focus. Journal of Consumer Research, 34(1), 57-65.

Keppel, G. (1982). Design and analysis: A researcher's handbook (2nd ed.). Englewood Cliffs, NJ: Prentice-Hall.

Lin, H. H., \& Wang, Y. S. (2006). An examination of the determinants of customer loyalty in mobile commerce contexts. Information Management, 43, 271-282.

Mantel, S. P., \& Kardes, F. R. (1999). The role of direction of comparison, attribute-based processing, and attitude-based processing in consumer preference. Journal of Consumer Research, 25(4), 335-352.

Morikawa, K., \& McBeath, M. K. (1992). Lateral motion bias associated with reading direction. Vision Research, 32(6), 1137-1141.

Preacher, K. J., \& Hayes, A. F. (2004). SPSS and SAS procedures for estimating indirect effects in simple mediation models. Behavior Research Methods, Instruments, \& Computers, 36, 717-731.

Rayner, K., Rotello, C. M., Stewart, A. J., Keir, J., \& Duffy, S. A. (2001). Integrating text and pictorial information: Eye movements when looking at print advertisements. Journal of Experimental Psychology. Applied, 7(3), 219-226.

Sanbonmatsu, D. M., Kardes, F. R., \& Gibson, B. D. (1991). The role of attribute knowledge and overall evaluations in comparative judgment. Organizational Behavior and Human Decision Processes, 48(1), 131-146.

Shiv, B., Edell, J. A., \& Payne, J. W. (1997). Factors affecting the impact of negatively and positively framed ad messages. Journal of Consumer Research, 24(3), 285-294.

Valenzuela, A., \& Raghubir, P. (2009). Position-based beliefs: The center-stage effect. Journal of Consumer Psychology, 19(2), 185-196. 